\title{
Cognitive and affective control in insomnia
}

\author{
Ralph E. Schmidt ${ }^{1,2}{ }^{*}$, Allison G. Harvey ${ }^{3}$ and Martial Van der Linden ${ }^{1,2}$ \\ 1 Department of Psychology, Swiss Center for Affective Sciences, University of Geneva, Geneva, Switzerland \\ 2 Department of Psychology, Cognitive Psychopathology and Neuropsychology Unit, University of Geneva, Geneva, Switzerland \\ ${ }^{3}$ Department of Psychology, University of California Berkeley, Berkeley, CA, USA
}

Edited by:

Gilles Pourtois, University of Ghent, Belgium

Reviewed by:

Giuseppe Di Pellegrino, University of Bologna, Italy

Nazanin Derakhshan, Birkbeck

College, UK

Chiara Baglioni, University of Freiburg

Medical Centre, Germany

${ }^{*}$ Correspondence:

Ralph E. Schmidt, Swiss Center for

Affective Sciences, University of

Geneva, Rue des Battoirs 7, CH-1205

Geneva, Switzerland.

e-mail: ralph.schmidt@unige.ch
Insomnia is a prevalent disabling chronic disorder. The aim of this paper is fourfold: (a) to review evidence suggesting that dysfunctional forms of cognitive control, such as thought suppression, worry, rumination, and imagery control, are associated with sleep disturbance; (b) to review a new budding field of scientific investigation - the role of dysfunctional affect control in sleep disturbance, such as problems with down-regulating negative and positive affective states; (c) to review evidence that sleep disturbance can impair nextday affect control; and (d) to outline, on the basis of the reviewed evidence, how the repetitive-thought literature and the affective science literature can be combined to further understanding of, and intervention for, insomnia.

\section{Keywords: affect, emotion regulation, insomnia, repetitive thought, rumination, thought control, thought suppression, worry}

\section{INTRODUCTION}

Insomnia is a prevalent disorder, with between 4 and $22 \%$ of people reporting chronic insomnia (Ancoli-Israel and Roth, 1999; Chevalier et al., 1999; Roth et al., 2011). The consequences of insomnia include complaints of impaired concentration and memory, elevated risk of accidents, more frequent use of medical services, and augmented work absenteeism (Ohayon et al., 1997; Roth and Ancoli-Israel, 1999). Furthermore, there is evidence that insomnia significantly heightens the risk of subsequently developing another psychiatric disorder, particularly an anxiety disorder, depression, or a substance-related disorder (Taylor et al., 2005; Johnson et al., 2006; Brower and Perron, 2010). Not surprisingly then, insomnia is regarded as a serious public health problem, with the direct and indirect costs associated with it in the United States estimated at between US $\$ 30$ and 35 billion annually (Chilcott and Shapiro, 1996).

Over the last decades, behavioral and cognitive approaches have led to significant advances in the etiology and treatment of this disorder (e.g., Harvey et al., 2005; Bootzin and Epstein, 2011). Cognitively inspired accounts of insomnia assume that mental hyperarousal (e.g., Harvey, 2002) or problems with mental dearousal (e.g., Espie, 2002) play a key role in acute and chronic forms of this disorder. In support of such a role, it has been found that poor sleepers are cognitively more aroused at bedtime than normal sleepers are (e.g., Robertson et al., 2007) and that poor sleepers perceive their "racing mind" as causally related to their sleep problems (e.g., Lichstein and Rosenthal, 1980). Cognitive accounts of insomnia also generally assume that inadequate emotional processing during the day will result in a surge of affect-laden concerns during the presleep period, thereby fueling excessive negatively toned cognitive activity (e.g., Espie, 2002; Harvey, 2002). However, as will be reviewed in more detail below, empirical research into affective aspects of sleep-interfering arousal is still in its early stages.
A growing line of research suggests that poor sleepers typically have difficulties relinquishing control when trying to fall asleep (Espie et al., 2006). Specifically, in the face of unwanted mental activity at bedtime, which can take the form of verbal thought or visual imagery (Harvey, 2000), poor sleepers tend to rely on dysfunctional control strategies that maintain cognitive and affective arousal instead of helping them to wind down (Harvey, 2002). In this article, we will first review evidence suggesting that dysfunctional forms of cognitive control, such as thought suppression, worry, rumination, and imagery control, are associated with sleep disturbance. We will then review more recent research suggesting that dysfunctional affect control, such as problems with downregulating negative and positive affective states, are also related to sleep disturbance. Moreover, we will review research indicating that sleep disturbance, in turn, can impair next-day affect control. Finally, we will outline how theoretical principles from the repetitive-thought literature could advance the study of cognitive control in insomnia, while principles and tools from the affective science literature could foster the study of affective control in insomnia. As will emerge from our analysis, a better understanding of the intricate interplay between cognitive and affective control could lead to the development of new intervention techniques that facilitate the transition to sleep.

\section{EXPLORATIONS OF THE ROLE OF COGNITIVE CONTROL IN INSOMNIA \\ EXPERIMENTAL STUDIES ON THE ROLE OF THOUGHT SUPPRESSION IN INSOMNIA}

Given that poor sleepers typically perceive their racing mind as causally related to their problems with sleep (e.g., Lichstein and Rosenthal, 1980), it seems inevitable that these individuals will do something in an attempt to prevent, modify, or suppress the thoughts that they perceive to be interfering with their sleep 
(Harvey, 2002). In this section, we will review evidence suggesting that ironic effects of thought suppression (e.g., Wegner, 1989; Najmi and Wegner, 2008) may be involved in the etiology and persistence of unwanted intrusive thoughts in insomnia.

Wegner (1994) accounted for the counterproductive effects of thought suppression by suggesting that the level of mental control enjoyed by an individual at any one time is a function of the joint action of a monitoring and an operating process. Termed the ironic process theory, it maintains that attempts to suppress involve (a) a controlled operating process that directs attention toward a distracter, that is, a thought other than the unwanted one; and (b) an automatic monitoring process that searches for failures to achieve the desired state and that, if necessary, renews the first process. In many circumstances, these interrelated processes work in tandem to successfully achieve suppression. However, there are a number of situations in which the balance is undermined, resulting in the unwanted thought intruding into consciousness. The "rebound" of the suppressed thought is explained by the fact that if the controlled distracter search is voluntarily relinquished or disabled by other resourcedemanding tasks, the automatic and therefore less resourcedependent monitoring lingers on, thereby enhancing the accessibility of the target thought (e.g., Najmi and Wegner, 2008). A series of studies highlight the importance of this theory to sleep and insomnia.

Harvey (2003) instructed individuals with insomnia and good sleepers either to suppress or to not suppress one issue/problem/thought while trying to get to sleep. On the night of the study, the participants who were told to suppress reported that they took longer to fall asleep and rated their sleep quality as poorer compared with participants given non-suppression instructions. This finding suggests that attempting to suppress a thought adversely affects both (a) self-reported sleep-onset latency and (b) sleep quality. The effect was detected for both the good sleepers and the patients with insomnia. Contrary to previous work (Wegner, 1989), there was no paradoxical increase in the frequency of the suppressed thought. However, it should be noted that the expected rebound of the suppressed thought might have occurred after sleep onset, which would have precluded it from emerging in the participants' reports about the presleep period. To test this hypothesis, Schmidt and Gendolla (2008) used an experimental design involving forced awakenings in a sleep laboratory. Half of the participants were instructed to suppress a target thought, whereas the other half freely thought of anything at all. Results revealed a reversal of target thought frequency at sleep onset: participants instructed to suppress reported fewer target thoughts than did controls before falling asleep, but more target thoughts afterward. In a related vein, three studies have found a suppression-induced increase of a target thought in dream mentation, especially if the target thought is laden with affect and if suppression is attempted under cognitive load (Wegner et al., 2004; Taylor and Bryant, 2007; Bryant et al., 2011). Although these studies on a "dream rebound" of suppressed thoughts did not assess sleep disturbance, they describe the mechanisms through which suppression of affect-laden content may entail a potentially sleep-interrupting rebound of the same content.

\section{EXPERIMENTAL STUDIES ON THE ROLE OF WORRY IN INSOMNIA}

Another strategy that is suggested to be commonly used by poor sleepers to manage unwanted thoughts at bedtime is worry (Borkovec, 1982; Watts et al., 1994). Research accumulated over several decades has shown that experimental manipulations designed to increase worry in good sleepers lengthen sleep-onset latency (Gross and Borkovec, 1982; Hall et al., 1996), whereas experimental manipulations designed to decrease worry in insomnia patients shorten sleep-onset latency (Haynes et al., 1981; Levey et al., 1991). An innovative set of investigations has been published more recently that confirms and extends these findings.

In one study, the approach to presleep worries was experimentally manipulated (Carney and Waters, 2006). Individuals with insomnia were asked either (a) to produce solutions to worries ("constructive worry") in the early evening, or (b) to list their worries and fill out worry questionnaires. The constructive worry group reported less cognitive arousal prior to bedtime and spent less time awake overall. These findings suggest that structured problem solving in the evening may reduce presleep cognitive arousal, thereby facilitating the transition to sleep.

In another recent study designed to test the relationship between sleeplessness and worry, undergraduate students grouped into high or low worriers on the basis of their responses on the Penn State Worry Questionnaire were given either $300 \mathrm{mg}$ caffeine (to induce sleeplessness) or a placebo prior to sleep (Omvik et al., 2007). Results indicated that high worriers did not report increased worry thoughts compared with low worriers in the face of sleeplessness. However, total sleep time as measured by actigraphy was reduced relatively more in high worriers than in low worriers as a consequence of caffeine-induced sleeplessness. This result might suggest that worries are comparatively more negative or intense and therefore more sleep-interfering in high worriers.

Recent research has also begun to explore the relationship between worry and rumination, which still needs to be clarified. Rumination has been defined as a "mode of responding to distress that involves repetitively and passively focusing on symptoms of distress and on the possible causes and consequences of these symptoms" (Nolen-Hoeksema et al., 2008). Worry and rumination are generally distinguished on the basis of their temporal orientation: worry refers to distress regarding future events, whereas rumination concerns thoughts of past events and current symptoms (e.g., Kaplan et al., 2009). In the first experimental investigation into the role of rumination for sleep, Guastella and Moulds (2007) examined the relations between rumination and sleep quality following a stressful midsemester exam in an undergraduate sample. Participants were asked either to ruminate about the exam ("think about how you felt when you were taking the test today") or to distract ("think about clouds forming in the sky") before sleep. The following morning, participants completed ratings of presleep intrusions about the exam and of sleep quality. Results indicated that although the rumination instruction led to more exam intrusions during the presleep period in participants with high and low trait rumination scores, only individuals with a trait tendency to ruminate reported reduced sleep quality following the rumination instruction. According to the authors, this finding suggests that soon after the rumination task was completed, low ruminators returned to their "default" cognitive 
style - that is, non-ruminative thought. In conjunction with correlational evidence that will be reviewed later (Carney et al., 2006, 2010), these results suggest that besides worry, rumination constitutes a form of cognitive control that may contribute to sleep disturbance.

\section{CORRELATIONAL STUDIES ON THE ROLE OF THOUGHT-CONTROL STRATEGIES IN INSOMNIA}

Apart from suppression, worry, and rumination, people may use a range of other thought-control strategies in an attempt to manage unwanted thoughts when trying to fall asleep. With the aim of exploring such strategies, Harvey (2001) adapted the ThoughtControl Questionnaire (TCQ), designed by Wells and Davies (1994), for use with patients with insomnia. This questionnaire, the TCQ-Insomnia (TCQ-I), was administered to 30 individuals with insomnia and to 30 good sleepers (Harvey, 2001). The results indicated that thought suppression, reappraisal and worry were used significantly more by participants with insomnia than by good sleepers. The use of suppression and worry is consistent with the experimental evidence discussed earlier. Regarding reappraisal, it may be an effective daytime strategy ensuring effective resolution of hassles and concerns, but it makes intuitive sense that engaging in similar processes at night might interfere with sleep onset.

In a follow-up study, a refined version of the TCQ-I was elaborated, which was termed the Thought-Control Questionnaire Insomnia-Revised (TCQI-R; Ree et al., 2005). The TCQI-R comprises six subscales: (1) aggressive suppression (e.g., "I get angry at myself for having the thought"); (2) cognitive distraction/suppression (e.g., "I think pleasant thoughts instead"); (3) behavioral distraction/suppression (e.g., "I try to block them out by reading, watching TV, or listening to the radio"); (4) social avoidance (e.g., "I avoid discussing the thought"); (5) worry (e.g., "I worry about more minor things"); and (6) reappraisal (e.g., "I try to reinterpret the thought"). A comparison between good sleepers and individuals with insomnia revealed that the latter more frequently used every thought-control strategy except for cognitive distraction. In accordance with the experimental evidence reviewed earlier, the strategies of aggressive suppression and worry proved particularly unhelpful, with the use of these techniques predicting poorer sleep quality in a sample that included good sleepers, subthreshold insomniacs, and insomniacs. In contrast, the use of cognitive distraction predicted better sleep quality. The validation study of the French version of the TCQI-R essentially replicated these findings (Schmidt et al., 2009): all six thoughtcontrol strategies were significantly related to facets of insomnia, with worry and aggressive suppression being most strongly related to insomnia symptoms and cognitive distraction functioning as a potential buffer against the latter.

In a recent extension of this line of research, a study with a large sample of young adults has revealed how certain personality traits may predispose people to rely on dysfunctional strategies of mental control when confronted with thoughts that keep them awake at night (Schmidt et al., 2010). Specifically, a structural equation model analysis indicated that individuals scoring high on two facets of impulsivity, namely, urgency and lack of perseverance, are particularly prone to respond with aggressive suppression and worry to unwanted mental activity at night. Impulsive urgency can be defined as the tendency to act rashly, especially under conditions of negative affect (Whiteside and Lynam, 2001). This facet has been shown to reflect a relative inability to deliberately inhibit dominant, automatic, or prepotent responses (Gay et al., 2008). As for lack of perseverance, it refers to an individual's inability to remain focused on a task, especially if the latter is boring or difficult (Whiteside and Lynam, 2001). This facet is linked to a relative inability to inhibit recurrent and irrelevant thoughts or memories (Gay et al., 2008).

The pathways through which the two mentioned facets of impulsivity may lead to an increase of unwanted cognitive activity at bedtime and thereby to a more intense use of dysfunctional control are twofold. Impulsive behavior may lead to a buildup of cognitive and affective arousal in the course of the day, which will then interfere with sleep and evoke more intense efforts at mental control. Additionally, or alternatively, problems with cognitive inhibition, as involved in lack of perseverance, may render individuals particularly vulnerable to unwanted intrusive thoughts at bedtime, and problems with behavioral inhibition, as involved in urgency, may prompt individuals to immediately do something about the unwanted thoughts, be it by way of mental or real-life responses. Below, we will review evidence for the first pathway, which suggests that impulsive urgency is indeed related to a surge of negative thoughts and emotions at bedtime (e.g., feelings of regret) that hinder the process of falling asleep (e.g., Schmidt and Van der Linden, 2009). The question of whether urgency and lack of perseverance also act on sleep-interfering mental activity through the second pathway remains to be explored.

Another important question awaiting further research is that of how dysfunctional thought-control may contribute to the transition from acute to chronic insomnia. In a pioneering study in this field, Jansson and Linton (2006) examined the associations between worry and poor sleep in relatively new (poor sleep 3-7 months) and chronic (poor sleep 8-12 months) insomnia sufferers. The main finding was that worry about sleeplessness was associated with poorer perceived sleep in individuals with chronic, but not recent, insomnia. The authors interpreted this finding as suggesting that the impact of sleep-related worry becomes increasingly stronger over time, which supports the idea that worry contributes to the maintenance of sleep problems and the development of chronic insomnia.

\section{STUDIES ON THE ROLE OF IMAGERY CONTROL IN INSOMNIA}

In parallel to the studies on thought control in insomnia, a literature has emerged that focuses on the control of imagery, that is, the ability to manage the occurrence of mental images. As described in the introduction, unwanted intrusive thoughts at bedtime can take the form of verbal thought or visual imagery. Borkovec et al. (1998) proposed that thinking about an emotional topic in verbal mode results in a drop in physiological response, which hinders in-depth processing and resolution of the emotional topic. Conversely, the translation of a concern into an image is suggested to increase physiological response in the short term, but will ultimately facilitate successful processing and the resolution of the emotion (Borkovec et al., 1998; Sibrava and Borkovec, 2006). Application of this theorizing to insomnia leads to the 
hypothesis that because presleep worry includes "the presence of active, picture-like images" (Coates et al., 1982), perhaps patients with insomnia spontaneously disengage from images to think about the same topic in verbal thought, thereby preventing emotional processing and contributing to the fueling of intrusive and worrisome thought (Harvey, 2002). Two published investigations have tested this proposal.

First, in an investigation of the imagery experienced during the presleep period in the natural home environment, 20 patients with insomnia and 20 good sleepers recorded when an image came to their mind by pressing a handheld counter (Nelson and Harvey, 2003). They then provided an oral description of the image and indicated whether the image was "pleasant," "unpleasant," or "neutral." This information was recorded on a voice-activated tape recorder. Analyses revealed that the insomnia group experienced more negatively valenced presleep images, but a lower number of images overall, relative to the good sleeper group. As negative imagery is likely to be associated with physiological and affective activation (Vrana et al., 1986), perhaps this activation motivates the quick and reflexive termination of images (hence the lower number of images overall reported by the insomnia group) in order to switch thought to the verbal mode.

Taking this line of investigation a step further, in an experimental manipulation of imagery control, individuals with insomnia were exposed to a stressor (speech threat) just prior to getting into bed and were instructed to think about the speech and its implications in either images (Image Group, $N=14$ ) or verbal thought (Verbal Group, $N=17$; Nelson and Harvey, 2002). Results indicated that in the short term, the Image Group reported more distress and arousal relative to the Verbal Group. In the longer term, the Image Group estimated that they fell asleep more quickly and, the following morning, reported less anxiety and more comfort about giving the speech compared with the Verbal Group. These findings are consistent with Borkovec et al.'s (1998) suggestion that the translation of negative-affect-laden concerns into imagery will ultimately facilitate emotional processing.

However, imagery may also be used in ways that divert attention from the processing of negative-affect-laden concerns and may in this way reduce unwanted presleep cognitive activity. In an experiment designed to test these assumptions (Harvey and Payne, 2002), individuals with insomnia were given one of three instructional sets to follow prior to sleep: instructions to distract using imagery, general instructions to distract, or no instructions. In the imagery distraction condition, participants were asked to distract themselves "by imagining a situation they found interesting and engaging, but also pleasant and relaxing." In support of the earlier mentioned assumptions, the imagery distraction group (a) reported shorter sleep-onset latency compared with the no instruction group and (b) rated their presleep thoughts, worries, and concerns as less uncomfortable and distressing than did the two other groups. Regarding the durability of this effect, the authors of the study note that although imagery distraction may be an effective short-term method to manage unwanted thoughts at bedtime, it may be less effective in the longer term because any "interesting and engaging" content will lose some of its attraction over time and will have to be replaced by new content.
In sum, the available evidence on imagery control and insomnia suggests that individuals with insomnia (a) may avoid negatively valenced imagery, which will hinder emotional processing and may thereby fuel negative cognitive activity at bedtime; and (b) may engage in positively valenced imagery to buffer negatively toned cognitive activity at bedtime, which may facilitate sleep in the short, but not long, term, unless the content of positive imagery is regularly renewed.

\section{AFFECTIVE TURN IN INSOMNIA RESEARCH}

As reviewed in the introduction, cognitive approaches to insomnia have led to the view that excessive mental activity at bedtime represents a central impediment to the process of falling asleep (e.g., Harvey, 2002). Although sleep-interfering mental activity is likely embedded in affective states, the latter have long received very little scientific attention. Recently, however, the importance of investigating affective processes in insomnia has been recognized (Harvey et al., 2009; Walker and Harvey, 2010) and a corresponding research agenda has been formulated (Baglioni et al., 2010a).

\section{STUDIES ON THE ROLE OF AFFECTIVE EXPERIENCE AND CONTROL IN INSOMNIA}

Initial empirical evidence for the role of emotion regulation came from two studies that investigated stress coping in good sleepers and insomniacs (Morin et al., 2003; LeBlanc et al., 2007). In both studies, stress coping skills were assessed with an inventory that distinguishes between three strategies, namely, task-oriented coping (e.g., coming up with several different solutions for a problem), emotion-oriented coping (e.g., blaming oneself for not knowing what to do), and avoidance-oriented coping (e.g., watching TV in order to distract oneself). Critically, it was found that patients with insomnia relied more often on emotion-oriented coping than good sleepers did, and that emotional coping increased perceived stress impact and presleep cognitive arousal, thereby contributing to sleep disturbance.

A number of other pioneering studies have in recent years investigated how specific affective states can impact sleep and how different control strategies may modify these affective states and their impact on sleep. In an extension of a line of research suggesting a negative impact of trait anger on sleep (Brissette and Cohen, 2002; Shin et al., 2005; Granö et al., 2008), StoiaCaraballo et al. (2008) examined the relations between forgiveness of interpersonal transgressions, anger rumination, and sleep quality in a sample of undergraduates by means of questionnaires. Using structural equation modeling, the authors found that forgiveness attenuated anger rumination and negative affect, thereby enhancing sleep quality.

Just as forgiving others seems to benefit sleep, forgiving oneself for one's own "wrongdoings" may also improve sleep, as a line of research on counterfactual emotions and sleep disturbance suggests. Such emotions include regret, shame, and guilt, which are termed counterfactual because they involve comparing the facts of one's own behavior with counterfactual imaginations of what one might or should have done (e.g., "If I had [not] done X, I would be in a better situation now"; e.g., Zeelenberg and Pieters, 2007). Initial hints at a negative impact of counterfactual emotions on 
sleep came from a questionnaire study by Wrosch et al. (2005), who found that regret intensity was associated with an omnibus measure of health problems, including chronic sleep problems, in a sample of older adults.

More recently, a series of studies have tested the idea that the time window prior to sleep might be particularly suitable for the emergence of counterfactual thoughts and emotions because bedtime is often the first quiet period available to review the day's events and one's own behavior. The results of an exploratory questionnaire study with undergraduate students lent initial support to this idea: counterfactual thoughts and emotions were often experienced at bedtime and their frequency was linked to selfreported insomnia severity (Schmidt and Van der Linden, 2009). Moreover, it was found that rash-action-prone students (with high scores on impulsive urgency) were particularly likely to engage in counterfactual processing at bedtime.

The findings obtained in the undergraduate sample (Schmidt and Van der Linden, 2009) were replicated and extended in a questionnaire study with elderly people (Schmidt et al., in press). In this study, participants were explicitly asked to indicate when in the course of the day they most often experienced regrets. The pattern of their responses clearly supported the idea that the presleep period is particularly suitable for the emergence of counterfactual emotions: while regret frequency remained at relatively low levels for most of the waking hours, a sharp rise occurred in the evening after going to bed. Moreover, analyses revealed that the nocturnal rise of regrets substantially contributed to insomnia severity independently of other well-known risk factors for latelife sleep disturbance, such as depression, sleep-interfering medical conditions, or medications. Finally, as with young adults, rashaction-prone older adults were particularly likely to experience regrets at bedtime and were therefore at a higher risk for sleep disturbances.

In the study with older adults (Schmidt et al., in press), participants were also asked to rate how often they relied on different thought-control strategies when trying to cope with their regrets at bedtime. It was found that the thought-control strategies of selfattacking (e.g., "I blame myself for having the regret"), thought suppression (e.g., "I try to stop thinking about the regret"), and worry (e.g., "Instead of the regret, I worry about more minor things") were positively associated with the frequency of nocturnal regrets and insomnia severity. These findings suggest that cognitive control may be exerted with the aim to achieve affective control, even if, as in the present study, the cognitive strategies turn out to be counterproductive.

Further evidence for sleep-interfering effects of counterfactual emotions has recently come from an experimental study by Schmidt and Van der Linden (in preparation). These authors asked a sample of undergraduate students to complete two questionnaires at home, one in the evening before going to bed, the other one in the morning after getting up. Participants were randomly assigned to one of three groups: prior to sleep, they were asked to describe the behavior they were most proud of, their most intense regret, or a standard working day (control condition). In the morning, they rated different dimensions of their sleep. In accordance with the earlier mentioned findings on counterfactual emotions and sleep (Schmidt and Van der Linden, 2009; Schmidt et al., in press), activation of preexisting regrets almost doubled the time taken to fall asleep in participants with high levels of habitual regrets when compared with the control condition.

With regard to the control of counterfactual emotions prior to sleep, the finding that self-attacking was closely associated with the frequency of nocturnal regrets and insomnia severity in the study by Schmidt et al. (in press) suggests that fostering selfforgiveness might also be beneficial for sleep, given that forgiving others for their wrongdoings has been shown to be beneficial for sleep quality (Stoia-Caraballo et al., 2008). Outside of the area of insomnia research, converging lines of evidence indicate that training in self-compassion or self-forgiveness may help down-regulate negative affective states that excessive self-criticism evokes (e.g., Ingersoll-Dayton and Krause, 2005; Gilbert and Procter, 2006).

An investigation by Wrosch et al. (2007, Study 2) provided preliminary evidence that regrets are amenable to intervention and that sleeping difficulties can in this way be reduced. In this study, participants were asked to report their most severe life regret and to assess its intensity twice with an interval of 3 months. At the same two time points, participants also rated their overall sleep quality during the past month. In the interim, they completed a writing intervention on three consecutive days. Participants in the experimental group were induced to engage in three regret-regulation strategies that have previously been shown to potentially serve a protective function: external attribution, downward social comparison, and disengagement from undoing the regret through selection of meaningful future goals (e.g., Heckhausen et al., 2010). Participants in the control group were instructed to write in a neutral, unemotional way about daily events and activities. Consistent with predictions, the authors found a general decline in regret intensity in the experimental group. Although the writing intervention did not show a main effect on changes in sleep problems, there was a significant interaction with initial regret intensity: participants in the experimental group who experienced high levels of regret intensity at the beginning of the study experienced fewer sleep problems over time, whereas the reverse was true of the participants in the control group.

Besides down-regulation of negative affective states, researchers have also begun to explore up-regulation of positive affective states for its effects on sleep. For instance, Steptoe et al. (2008) found in a large sample of civil servants that both hedonic well-being (positive affect), as captured by ecological momentary assessment, and eudaimonic well-being (purposeful engagement with life) were inversely related to sleep problems after adjustment for age, gender, household income, and self-rated health. Of note, hedonic and eudaimonic well-being dampened adverse effects of a number of negative psychosocial factors on sleep, including financial strain, social isolation, low emotional support, negative social interactions, and psychological distress. However, as the authors of the study emphasize, the relations between sleep and affect are likely bidirectional and the cross-sectional design of their investigation did not allow them to draw causal inferences.

Two groups of researchers have investigated how trait and state gratitude may influence affect and sleep, complementary to these findings on well-being and sleep. Emmons and McCullough (2003, Study 3 ) asked a sample of people with neuromuscular disease to complete "daily experience rating forms" in the early evening for 
3 weeks. In these forms, participants rated, among other variables, their daily affect, subjective well-being, and sleep. Unbeknownst to the participants, they were assigned to one of two experimental conditions. In the control condition, they simply completed the mentioned forms. In the gratitude condition, they were asked to count their blessings, that is, to write down up to five things that they were grateful or thankful for. Analyses revealed that the gratitude induction increased positive affect, reduced negative affect, and improved sleep quantity and quality. Pursuing this line of research, Wood et al. (2009) examined the relations between trait gratitude and sleep in a cross-sectional questionnaire study with a large community sample. The authors found that trait gratitude predicted greater subjective sleep quantity and quality, shorter sleep latency, and less daytime dysfunction. Moreover, the relation between gratitude and each of the sleep variables was mediated by more positive and fewer negative presleep cognitions.

Although these studies seem to clearly suggest that upregulation of positive affect is beneficial for sleep, some recent findings caution against too simplistic a view that would equate positive affect with good sleep and negative affect with bad sleep. For instance, Talbot et al. (2009) examined the effects of mood induction on sleep in a group of individuals with interepisode bipolar disorder and in a group of healthy controls. Participants spent two baseline nights in the sleep laboratory, followed by a happy mood induction night and a sad mood induction night. Results indicated a significant interaction whereby on the happy mood induction night, the bipolar group exhibited significantly longer sleep-onset latency than did the control group, whereas there was no difference on the baseline nights. Moreover, the bipolar group exhibited a (non-significantly) longer sleep-onset latency on the happy mood induction night compared with the baseline night, whereas the control group exhibited significantly shorter sleep-onset latency on the happy mood induction night. On the sad mood induction night, participants in both groups had shorter sleep-onset latency. These findings suggest that problems with the regulation of positive affect may contribute to sleep-onset insomnia in bipolar individuals, possibly because these individuals reacted to the happy mood induction with an activating focus on the pursuit of goals and rewards (cf. Johnson, 2005).

\section{STUDIES ON THE ROLE OF SLEEP FOR AFFECTIVE EXPERIENCE AND CONTROL}

Up to this point, we have considered how dysfunctions in affective control may adversely impact sleep. In addition, accumulating evidence suggests that lack of sleep, in turn, may adversely impact next-day affect. Thus, the relations between sleep and affect are best conceptualized as bidirectional (Walker and Harvey, 2010), with the potential of negative effects escalating into a vicious circle. For instance, Buysse et al. (2007) used ecological momentary assessment to obtain ratings of daytime symptoms from insomnia patients. Results indicated that, when compared with good sleepers, individuals with insomnia endorsed higher negative mood and lower positive mood. More recently, Baglioni et al. (2010b) presented blocks of neutral, negative, positive, sleep-related negative, and sleep-related positive pictures to individuals with primary insomnia and good sleepers. During the presentation, facial electro-myography and -cardiography were performed. Analyses revealed that individuals with primary insomnia exhibited greater inhibition of the corrugator activity in response to sleep-related positive stimuli as compared with the other blocks of stimuli, suggesting a heightened motivation and sensitivity toward good sleep. At the same time, individuals with insomnia rated sleeprelated negative stimuli as more unpleasant and arousing and showed higher cardiac vagal tone in response to all stimuli as compared with good sleepers, suggesting subjective hyperarousal in the context of poor sleep and a general physiological hyperarousal.

Furthermore, converging lines of clinical evidence suggest that sleeping difficulties adversely impact affective disorders (including anxiety, depression, and bipolar disorder) and problems with anger, aggression, and impulse control, especially among children and adolescents (e.g., Jenni and Dahl, 2008). For example, in patients with bipolar disorder, the most common prodrome of mania is sleep disturbance (Jackson et al., 2003). And with regard to sleep and aggression, Haynes et al. (2006), for instance, examined the effects of a 6-week behavioral sleep intervention in adolescents who had recently been treated for substance abuse. It was found that increases in sleep time were associated with decreases in aggressive ideation and aggressive actions occurring during conflicts. Taken together, the available clinical evidence thus clearly suggests that poor sleep impairs affective and behavioral control - and that these effects may be reversed through sleep-promoting interventions.

Additional evidence for effects of sleep on next-day affect comes from sleep-deprivation studies. However, these studies must be interpreted with the caveat that findings from sleep-deprived normal sleepers may not readily be extrapolated to chronic poor sleepers because, among other reasons, insomnia involves adaptation processes and is not necessarily associated with reduced sleep quantity. In a recent sleep-deprivation study, Talbot et al. (2010) investigated the impact of experimentally shortened sleep in adolescents and adults. The main findings were that sleepdeprivation reduced positive affect and increased anxiety during a "catastrophizing task," in which an interviewer repeatedly asked questions about each participant's most threatening worry (for details, see Vasey and Borkovec, 1992). Analogous results have been reported for work-related sleep deprivation. For example, Zohar et al. (2005) investigated the longitudinal relations between night-shift-related sleep loss and emotional reactivity in medical residents. Analyses of ecological momentary assessment and actigraphic sleep data indicated that sleep loss intensified negative emotions following goal-disruptive daytime events, whereas positive emotions were attenuated following goal-conducive daytime events. Increased reactivity to negative stimuli following sleep deprivation has also been found in an experimental study with healthy young adults (Franzen et al., 2009): when compared with a normal sleep control group, the sleep-deprived group displayed a larger pupil diameter when viewing negative pictures.

In the first study that used functional brain imaging to investigate the neural underpinnings of affective dysregulation following sleep loss (Yoo et al., 2007), sleep-deprived individuals showed a $60 \%$ increase of amygdala activation in response to increasingly negative stimuli from the International Affective Picture System, a threefold increase in the extent of amygdala volume that was activated, and a loss of functional connectivity between the amygdala 
and the medial-prefrontal cortex. This brain region exerts topdown control on the limbic area (including the amygdala) and regulates emotional responses to render them appropriate for the context. The study thus suggests that sufficient sleep is indispensable for maintaining prefrontal control over affective reactions and behavior (see also Walker and van der Helm, 2009).

\section{TOWARD AN INTEGRATIVE VIEW OF COGNITIVE AND AFFECTIVE CONTROL IN INSOMNIA}

As argued throughout this article, the stream of consciousness in the transition to sleep may be decomposed into cognitive aspects, such as thoughts and images, and affective aspects, such moods and emotions. Recent accounts of insomnia have proposed that both cognitive and affective aspects of mental hyperactivity at bedtime may contribute to sleeping difficulties (e.g., Espie, 2002; Harvey, 2002). However, as may be gathered from the preceding review of the literature, research has as yet mainly focused on cognitive aspects of sleep-interfering mental activity and only just begun to empirically explore affective aspects. In what follows, we will describe how an integrative view of cognitive and affective control in the transition to sleep could advance our understanding of insomnia and lead to the development of new prevention and intervention techniques.

\section{NEW INSIGHTS FROM THE REPETITIVE-THOUGHT LITERATURE}

Regarding cognitive control in insomnia, converging lines of correlational and experimental evidence suggest that two forms of thought control that are often used by poor sleepers are particularly dysfunctional, namely, thought suppression and worry. However, a question calling for conceptual and empirical clarification is that of the relation between worry and rumination. Rumination has also been found to be associated with poor sleep (Carney et al., 2006; Guastella and Moulds, 2007), and one recent study has suggested that rumination may in important ways contribute to clinical insomnia independently of worry and depressed mood states (Carney et al., 2010). In a similar vein, an earlier questionnaire study with students had revealed that rumination was associated with subjective sleep quality even after controlling for negative mood (Thomsen et al., 2003). Of importance, these findings suggest that problems with cognitive control may contribute to sleep problems independently of negative mood states.

To clarify the relation between worry and rumination in the context of insomnia, some researchers have suggested conceptualizing them as two forms of repetitive thought (e.g., Carney et al., 2010). Repetitive thought has come to be seen as an important perpetuating factor that pervades a wide range of psychological disorders (e.g., Harvey et al., 2004). Outside of the area of insomnia research, several researchers have in recent times proposed comprehensive approaches to repetitive thought that encompass worry and rumination (e.g., Segerstrom et al., 2003; Watkins, 2008). Of note, these approaches assume that repetitive thought is not necessarily unconstructive: depending on its characteristics, it may take constructive forms, such as planning and problem solving. This assumption accords with the earlier reviewed evidence that structured problem solving in the evening may reduce presleep cognitive arousal, thereby facilitating sleep (Carney and Waters, 2006).
According to Watkins (2008), three main features of repetitive thought account for differential outcomes: (a) the valence of the thought content, (b) the intrapersonal and situational context in which repetitive thought occurs, and (c) the level of construal (abstract vs. concrete processing). As to valence, a majority of studies have, unsurprisingly, found that repetitive thought amplifies negatively valenced content, thereby leading to unconstructive outcomes. However, as Watkins (2008) pointed out, positive content, when excessively repeated, may also lead to unconstructive outcomes, for example, in individuals vulnerable to hypomania and mania. This consideration is of particular importance in the context of insomnia because, as reviewed earlier, positive mood may contribute to sleep-onset insomnia, at least in certain clinical conditions, such as bipolar disorder (Talbot et al., 2009). For the "embeddedness" of repetitive thought, both the intrapersonal and the situational context can range from negatively valenced (e.g., intrapersonal: dysphoric mood, negative expectations, low self-esteem; situational: stressful, traumatic events) to positively valenced (intrapersonal: positive mood, positive expectations, high self-esteem; situational: successful, rewarding events). The available empirical evidence suggests that the prevailing valence of an individual's cognitive-affective system largely determines whether repetitive thought is helpful or unhelpful (Watkins, 2008). For instance, lack of self-esteem and self-criticism have been shown to moderate the effect of rumination on mood and cognition (e.g., Robinson and Alloy, 2003) - findings that are of direct relevance for insomnia because, as reported earlier, self-criticism and self-attacking have been associated with disturbed sleep in a number of studies (e.g., Ree et al., 2005; Schmidt et al., 2009; Vincent et al., 2009).

Although valence, as mentioned earlier, is a major factor in determining the consequences of repetitive thought, it cannot explain all empirical findings. For instance, repetitive thought focused on negative content has been found to have constructive consequences in some studies of depressive rumination and of defensive pessimism. Moreover, simply focusing on positive content is not necessarily the most constructive form of repetitive thought, as revealed by research on process and outcome simulations (Watkins, 2008). To account for the available evidence, Watkins (2008) therefore proposed level of construal as a third main characteristic of repetitive thought and hypothesized that, at least when repetitive thought is focused on negative content, low-level, more concrete construals have more constructive consequences than do high-level, more abstract construals (see also Watkins, 2011). Regarding the mechanisms by which level of construal may influence the consequences of repetitive thinking, Watkins (2008) mentions that lower level construals should provide more elaborated and contextual detail about means, alternatives, and actions, thereby promoting problem solving in difficult, novel, or complex situations. Moreover, concrete construals may facilitate self-regulation by focusing attention on the immediate demands, reducing anxiety, and requiring less effort and thus consuming fewer resources. Finally, in negative situations, more abstract construals could provoke negative overgeneralizations in the sense that a single failure is interpreted in terms of individual inadequacy (e.g., "I am a failure") rather than in terms of situational difficulties (e.g., "I failed this hard exam"). Indirect evidence for 
the relevance of level of construal for sleep-interfering mentation has recently come from studies suggesting that mindfulness-based cognitive therapy (MBCT), which uses meditation techniques to train patients away from abstract levels of processing and into more concrete modes of processing (e.g., Carney and Segal, 2005), may be beneficial for sleep (e.g., Ong et al., 2008).

In sum, the literature on repetitive thought clearly suggests that it might be promising to conceptualize presleep worry and rumination within a larger theoretical framework and to examine sleep-interfering mental activity along the dimensions of valence, intrapersonal and situational context, and level of construal. In particular, such a conceptualization may help elucidate the relations between thought control, imagery control, and affective control in the context of insomnia. For instance, worry comprises mostly negatively valenced verbal thoughts, rather than visual images, and is more abstract and less concrete than other types of mental processing (Sibrava and Borkovec, 2006). The avoidance of more concrete imagery in worry is postulated to serve a protective function, whereby physiological activation and emotional processing are reduced at the expense of maintaining anxious meanings. A promising avenue for future research will be to determine whether promoting more concrete levels of construal, for example, by means of MBCT or exposure therapy, may foster imagery-related emotional processing in worry, thereby improving sleep. Another fruitful line of inquiry will be to investigate the links between suppression and different forms of repetitive thought, as exemplified in the study by Watkins and Moulds (2009), in the context of insomnia.

\section{NEW INSIGHTS FROM THE AFFECTIVE SCIENCE LITERATURE}

In this section, we will suggest that the theoretical framework for repetitive thought as elaborated by Watkins (2008) can also serve as a conceptual platform to investigate affective control in insomnia. As detailed in the previous section, Watkins' framework incorporates affective states in two ways: (a) by taking into account thought valence and (b) by taking into account subjective affective states (e.g., negative mood) within the intrapersonal context. However, it should be underscored that Watkins advocates a clear distinction between cognitive and affective aspects of repetitive thought at the conceptual level and recommends the development and use of repetitive-thought questionnaires that do not confound repetitive thought with the degree of negative affectivity.

As in the case of repetitive thought, the question naturally arises as to which dimensions of affective states may impact sleep. In a first empirical attempt to uncover sleep-relevant dimensions of affective states, Stewart et al. (2011) asked a sample of students to complete questionnaires assessing depressive symptoms, trait anxiety, trait anger, trait positive affect, trait rumination, and sleep quality. Structural equation modeling analyses revealed that negative affect (the shared variance among the measures of depression, trait anxiety, and trait anger) was related to poor sleep, whereas no unique effects were observed for any of the negative and positive affective factors. Of note in the present context, the relation between negative affect and sleep was independent of rumination. The latter finding suggests that affective factors can influence sleep in ways that are separable from the effects of cognitive hyperactivity, thereby highlighting the added value of an affective science approach to insomnia.

On the basis of their findings, Stewart et al. (2011) concluded that "... the shared, not unique, aspects of negative emotional factors may be key determinants of sleep quality" (p. 609). However, a limitation of their study resides in the fact that it almost exclusively used trait measures. Thus, certain transient affective states of negative valence, such as regrets (e.g., Schmidt and Van der Linden, in preparation), or of positive valence, such as gratitude (e.g., Emmons and McCullough, 2003), may well have unique effects on sleep. Of note, induction of regrets prior to sleep delayed sleep onset independently of levels of depressed mood, as assessed by the Beck Depression Inventory-II (Beck et al., 1996), in the study by Schmidt and Van der Linden (in preparation). Moreover, positive affective states may have opposite effects on sleep in normal and certain clinical populations, such as individuals with bipolar disorder (e.g., Talbot et al., 2009). In addition, as the study by Talbot and colleagues also revealed, specific affective states may influence certain sleep parameters but not others (e.g., sleep-onset latency vs. rapid eye movement density). Clearly, more research is needed to unravel the associations between the various dimensions of affective states and subjective as well as objective sleep parameters in normal and clinical populations.

Apart from valence, arousal, which may be further differentiated into bodily excitation and intensity of feeling, is another fundamental dimension of affective states (e.g., Scherer, 2005). For instance, intense anger is likely characterized by high bodily excitation, whereas intense sadness may be accompanied by very low bodily activation. A better understanding of affective arousal in the context of insomnia is all the more important as this dimension may account for findings suggesting that both positive and negative affect may impair sleep (e.g., Talbot et al., 2009). Regarding the assessment of presleep arousal, research has as yet mainly focused on the cognitive and somatic dimensions. For example, Nicassio et al.'s (1985) Presleep Arousal Scale comprises a cognitive arousal and a somatic arousal subscale; more recently, a refined instrument to assess presleep cognitive arousal has been developed (Harvey and Espie, 2004). However, to the best of our knowledge, no instrument that specifically captures presleep affective arousal in its two subdimensions is currently available. Yet, outside of insomnia research, recent advances in affective sciences have led to the development of new assessment tools that could be adapted for investigations of sleep. For example, the Geneva Emotion Wheel (Scherer, 2005) allows one to rate the valence and intensity of a wide range of emotions by checking corresponding circles in a graphical display.

A further complication arises from the fact that, in the area of insomnia research, the terms "emotion" and "affect" have often been used interchangeably although the two concepts should be clearly distinguished. Within the field of affective sciences, emotions are generally considered as one particular type of affective state that features the following components (e.g., Scherer, 2005): (a) a cognitive component (appraisals); (b) a subjective feeling component (emotional experience); (c) a neurophysiological component (bodily symptoms); (d) a motor expression component (vocal, facial, and postural display); and (e) a motivational component (action tendencies). As compared with affect 
dispositions (e.g., hostility) and moods (e.g., depressed mood), which have often been investigated in connection with sleep, emotions are: (a) typically linked to a stimulus event, (b) of shorter duration, and (c) of greater intensity. In view of their greater intensity, emotions deserve special scientific attention in the context of insomnia. As reviewed earlier, a number of recent investigations have provided evidence for sleep-interfering effects of specific emotions, such as regret, shame, and guilt (e.g., Schmidt and Van der Linden, 2009; Schmidt et al., in press). Given that emotions are endowed with specific action tendencies, emotions might have unique sleep-interfering effects that are not accounted for by general effects of valence or arousal. For instance, anger comprises action tendencies of opposition or assault (e.g., Frijda et al., 1989), whereas regret is typically accompanied by such action tendencies as to "kick oneself" and to correct one's mistake (e.g., Berndsen et al., 2004). Obviously, such action tendencies, if experienced at bedtime, have a strong potential to interfere with the process of falling asleep, especially in impulsive individuals with actioninhibition difficulties (cf. Schmidt and Van der Linden, 2009). Exploration of sleep-interfering action tendencies in the context of affective control might thus be a worthy endeavor.

Analysis of presleep mental activity in terms of these emotion components reveals yet another underinvestigated area in the context of affective control and insomnia, namely, the effects of (non-)expression of emotions. In a pioneering study in this field, Caska et al. (2009) found in a large sample of patients with coronary heart disease that self-rated suppression of anger expression was substantially associated with poor sleep quality. This association remained strong even after adjusting for demographics, comorbidities, lifestyle factors, medications, cardiac function, depressive symptoms, and trait anger. Apart from cognitive control strategies, such as thought suppression and worry, behavioral forms of affect control may thus have an important impact on sleep, which clearly calls for further investigation.

Also underinvestigated in the context of insomnia is what Gross and Thompson (2007) termed "antecedent-focused emotion regulation.” This term refers to anticipatory forms of regulation that intervene before a full-blown emotional response occurs. For instance, an individual may selectively approach situations that are likely to evoke positive emotions or avoid situations that are liable to provoke negative emotions. To date, most studies in clinical research have focused on "response-focused emotion regulation," that is, retrospective forms of regulation, such as the cognitive strategies of thought suppression and worry, which are typically used to deal with emotions once they have occurred. In the first study to investigate the relations between antecedentfocused emotion regulation and sleep, Schmidt and Van der Linden (2011) hypothesized that individuals who have difficulties anticipating potential future regrets are at an increased risk of blindly running into situations or adopting behaviors that are prone to evoke regrets. As a consequence, they should experience more actual regrets and related counterfactual emotions, which have been shown to interfere with sleep (Schmidt and Van der Linden, 2009; Schmidt et al., in press). To test these ideas, Schmidt and Van der Linden (2011) developed the Regret Anticipation Failures Scale and administered it, within a battery of other questionnaires, to university students. Results supported the predictions mentioned: regret anticipation failures in the course of the day were associated with the frequency of regret-related counterfactual thoughts and emotions at bedtime, and the latter mediated the effect of regret anticipation failures on self-rated insomnia severity. These preliminary findings encourage further investigation of the implications of antecedent-focused emotion regulation for sleep. For instance, an intriguing question is that of whether training in affect anticipation might help people avoid negative emotional incidents in the course of the day, thereby obviating the need to engage in response-focused emotion regulation (e.g., thought suppression, worry) at the end of the day.

\section{SUMMARY AND OUTLOOK}

In the first part of this article, we reviewed a coherent body of evidence suggesting that dysfunctional forms of cognitive control, such as thought suppression, worry, rumination, and imagery control, are associated with sleep disturbance, as they are with other forms of psychopathology. In the second part of this article, we presented more recent lines of evidence indicating that dysfunctional forms of affective control, such as problems with down-regulating negative and positive affective states, are also related to sleep disturbance, and that sleep disturbance, in turn, can impair next-day affect control. In the third part of this article, we outlined how principles from the repetitive-thought literature and the affective science literature can inspire new lines of investigation and help develop a theoretical framework that integrates the roles of cognitive and affective control in insomnia.

An important theoretical question that calls for further research is that of whether dysfunctional forms of cognitive and affective control may be viewed as separate, though related, mechanisms, or may rather stem from a unitary, core mechanism. As reviewed earlier, some of the available suggests that dysfunctional forms of cognitive control, such as thought suppression, are often used to down-regulate negative affective states, for example regrets (e.g., Schmidt et al., in press). However, there is also evidence suggesting that cognitive factors and affective factors may independently impact sleep. For instance, in the previously mentioned study by Stewart et al. (2011), the relation between negative affect (as captured by the shared variance among measures of depression, trait anxiety, and trait anger) and sleep was independent of rumination. Conversely, another study has revealed that rumination may in important ways contribute to clinical insomnia independently of depressed mood states (Carney et al., 2010). Taken together, these preliminary findings suggest that affective factors might contribute to insomnia over and above the influence of well-established cognitive factors.

Regarding methodology, a limitation of the reviewed literatures on cognitive and affective control in insomnia resides in the fact that most studies have as yet relied on self-report. However, conclusions from these investigations continue to be strengthened and extended by the use of behavioral measures, including actigraphy (e.g., Omvik et al., 2007), physiological measures (e.g., Baglioni et al., 2010b), functional brain imaging (e.g., Yoo et al., 2007), and clinical intervention studies (e.g., Haynes et al., 2006). Clearly, more research with these methods is needed to advance our understanding of the interplay between cognitive and affective factors in insomnia. 
With respect to the treatment of insomnia, the reviewed literatures contain a number of indications of how the standard cognitive-behavioral therapy (e.g., Bootzin and Epstein, 2011) could be complemented with interventions that specifically address dysfunctional forms of cognitive and affective control. For instance, training in structured problem solving might counteract non-constructive forms of repetitive-thought prior to sleep (e.g., Carney and Waters, 2006), or training in self-compassion (e.g., Gilbert and Procter, 2006) might defuse sleep-interfering effects

\section{REFERENCES}

Ancoli-Israel, S., and Roth, T. (1999). Characteristics of insomnia in the United States: results of the 1991 National Sleep Foundation Survey I. Sleep 22, S347-S353.

Baglioni, C., Lombardo, C., Bux, E., Hansen, S., Salveta, C., Biello, S., Violani, C., and Espie, C. A. (2010a). Psychophysiological reactivity to sleep-related emotional stimuli in primary insomnia. Behav. Res. Ther. 48, 467-475.

Baglioni, C., Spiegelhalder, K., Lombardo, C., and Riemann, D. (2010b). Sleep and emotions: a focus on insomnia. Sleep Med. Rev. 14, 227-238.

Beck, A. T., Steer, R. A., and Brown, G. K. (1996). Manual for the Beck Depression Inventory, 2nd Edn, San Antonio, TX: The Psychological Corporation.

Berndsen, M., van der Pligt, J., Doosje, B., and Manstead, A. S. R. (2004). Guilt and regret: the determining role of interpersonal and intrapersonal harm. Cogn. Emot. 18, 55-70.

Bootzin, R. R., and Epstein, D. R. (2011). Understanding and treating insomnia. Annu. Rev. Clin. Psychol. 7, 435-458.

Borkovec, T. D. (1982). Insomnia. J. Consult. Clin. Psychol. 50, 880-895.

Borkovec, T. D., Ray, W. J., and Stöber, J. (1998). Worry: a cognitive phenomenon linked to affective, physiological, and interpersonal behavioural processes. Cognit. Ther. Res. 22, 561-576.

Brissette, I., and Cohen, S. (2002). The contribution of individual differences in hostility to the associations between daily interpersonal conflict, affect, and sleep. Pers. Soc. Psychol. Bull. 28, 1265-1274.

Brower, K. J., and Perron, B. E. (2010). Sleep disturbance as a universal risk factor for relapse in addictions to psychoactive substances. Med. Hypotheses 74, 928-933.

Bryant, R. A., Wyzenbeek, M., and Weinstein, J. (2011). Dream rebound of suppressed emotional thoughts: the influence of cognitive load. Conscious. Cogn. 20, 515-522.
Buysse, D. J., Thompson, W., Scott, J., Franzen, P. L., Germain, A., Hall, M., Moul, D. E., Nofzinger, E. A., and Kupfer, D. J. (2007). Daytime symptoms in primary insomnia: a prospective analysis using ecological momentary assessment. Sleep Med. 8, 198-208.

Carney, C. E., Edinger, J. D., Meyer, B., Lindman, L., and Istre, T. (2006). Symptom-focused rumination and sleep disturbance. Behav. Sleep Med. 4, 228-241.

Carney, C. E., Harris, A. L., Moss, T. G., and Edinger, J. D. (2010). Distinguishing rumination from worry in clinical insomnia. Behav. Res. Ther. $48,540-546$.

Carney, C. E., and Segal, Z. V. (2005). "Mindfulness based cognitive therapy," in Innovations in Clinical Practice: Focus on Adults and Families, ed. L. VandeCreek (Sarasota: Professional Resource Press), 5-18.

Carney, C. E., and Waters, W. F. (2006). Effects of a structured problemsolving procedure on pre-sleep cognitive arousal in college students with insomnia. Behav. Sleep Med. 4, 13-28.

Caska, C. M., Hendrickson, B. E., Wong, M. H., Ali, S., Neylan, T., and Whooley, M. A. (2009). Anger patients with coronary heart disease: findings from the heart and soul study. Psychosom. Med. 71, 280-285.

Chevalier, H., Los, F., Boichut, D., Bianchi, M., Nutt, D. J., Hajak, G., Hetta, J., Hoffmann, G., and Crowe, C. (1999). Evaluation of severe insomnia in the general population: results of a European multinational survey. J. Psychopharmacol. 13, S21-S24.

Chilcott, L. A., and Shapiro, C. M. (1996). The socioeconomic impact of insomnia: an overview. Pharmacoeconomics 10(Suppl. 1), 1-14.

Coates, T. J., Killen, J. D., George, J., Marchini, E., Silverman, S., and Thoresen, C. (1982). Estimating sleep parameters: a multitraitmultimethod analysis. J. Consult. Clin. Psychol. 50, 345-352. expression and sleep quality in

of self-attacking (e.g., Schmidt et al., in press). These techniques exemplify how basic research into cognitive and affective control in insomnia may ultimately translate into new forms of intervention.

\section{ACKNOWLEDGMENTS}

This research was supported by (a) the National Center of Competence in Research (NCCR) in Affective Sciences financed by the Swiss National Science Foundation (51NF40-104897) and hosted by the University of Geneva, and (b) NIMH Grant R34 MH080958.

Emmons, R. A., and McCullough, M. E. (2003). Counting blessings versus burdens: an experimental investigation of gratitude and subjective wellbeing in daily life. J. Pers. Soc. Psychol. 84, 377-389.

Espie, C. A. (2002). Insomnia: conceptual issues in the development, persistence, and treatment of sleep disorders in adults. Annu. Rev. Psychol. 53, 215-243.

Espie, C. A., Broomfield, N. M. MacMahon, K. M. A., Macphee, L. M., and Taylor, L. M. (2006). The attention-intention-effort pathway in the development of psychophysiologic insomnia: a theoretical review. Sleep Med. Rev. 10, 215-245.

Franzen, P. L., Buysse, D. J., Dahl, R. E., Thompson, W., and Siegle, G. J. (2009). Sleep deprivation alters pupillary reactivity to emotional stimuli in health young adults. Biol. Psychol. 80, 300-305.

Frijda, N. H., Kuipers, P., and ter Schure, E. (1989). Relations among emotion, appraisal, and emotional action readiness. J. Pers. Soc. Psychol. 57, 212-228.

Gay, P., Rochat, L., Billieux, J., d'Acremont, M., and Van der Linden, M. (2008). Heterogeneous inhibition processes involved in different facets of self-reported impulsivity: evidence from a community sample. Acta Psychol. (Amst.) 129, 332-339.

Gilbert, P., and Procter, S. (2006). Compassionate mind training for people with high shame and self-criticism: overview and pilot study of a group therapy approach. Clin. Psychol. Psychother. 13, 353-379.

Granö, N., Vahtera, J., Virtanen, M., Keltikangas-Järvinen, L., and Kivimäki, M. (2008). Association of hostility with sleep duration and sleep disturbances in an employee population. Int. J. Behav. Med. 15, 73-80.

Gross, J. J., and Thompson, R. A. (2007). "Emotion regulation: conceptual foundations," in Handbook of Emotion Regulation, ed. J. J. Gross (New York: Guilford Press), 3-24.
Gross, R. T., and Borkovec, T. D. (1982). The effects of a cognitive intrusion manipulation on the sleep onset latency of good sleepers. Behav. Ther. 13, 112-116.

Guastella, A. J., and Moulds, M. L. (2007). The impact of rumination on sleep quality following a stressful life event. Pers. Individ. Dif. 42, 1151-1162.

Hall, M., Buysse, D. J., Reynolds, C. F. Kupfer, D. J., and Baum, A. (1996). Stress-related intrusive thoughts disrupt sleep-onset and continuity. Sleep Res. 25, 163.

Harvey, A. G. (2000). Pre-sleep cognitive activity: a comparison of sleep-onset insomniacs and good sleepers. Br. J. Clin. Psychol. 39, 275-286.

Harvey, A. G. (2001). I can't sleep, my mind is racing! An investigation of strategies of thought control in insomnia. Behav. Cogn. Psychother. 29, 3-11.

Harvey, A. G. (2002). A cognitive model of insomnia. Behav. Res. Ther. 40, 869-893.

Harvey, A. G. (2003). The attempted suppression of presleep cognitive activity in insomnia. Cognit. Ther. Res. 27, 593-602.

Harvey, A. G., McGlinchey, E., and Gruber, J. (2009). "Toward an affective science of insomnia treatments," in Emotion Regulation and Psychopathology: A Transdiagnostic Approach to Etiology and Treatment, eds A. M. Kring and D. S. Sloan (New York: Guilford Press), 427-446.

Harvey, A. G., and Payne, S. (2002) The management of unwanted presleep thoughts in insomnia: distraction with imagery versus general distraction. Behav. Res. Ther. 40, 267-277.

Harvey, A. G., Tang, N. K. Y., and Browning, L. (2005). Cognitive approaches to insomnia. Clin. Psychol. Rev. 25, 593-611.

Harvey, A. G., Watkins, E., Mansell, W., and Shafran, R. (2004). Cognitive Behavioural Processes across Psychological Disorders: A Transdiagnostic Approach to Research and Treatment. Oxford: Oxford University Press. 
Harvey, K., and Espie, C. A. (2004). Development and preliminary validation of the Glasgow Contents of Thoughts Inventory (GCTI): a new measure for the assessment of presleep cognitive activity. $\mathrm{Br}$. J. Clin. Psychol. 43, 409-420.

Haynes, P. L., Bootzin, R. R., Smith, L., Cousins, J., Cameron, M., and Stevens, S. (2006). Sleep and aggression in substance-abusing adolescents: results from an integrative behavioral sleep-treatment program. Sleep 29, 512-520.

Haynes, S. N., Adams, A., and Franzen, M. (1981). The effects of presleep stress on sleep-onset insomnia. J. Abnorm. Psychol. 90, 601-606.

Heckhausen, J., Wrosch, C., and Schulz, R. (2010). A motivational theory of life-span development. Psychol. Rev. 117, 32-60.

Ingersoll-Dayton, B., and Krause, N. (2005). Self-forgiveness: a component of mental health in later life. Res. Aging 27, 267-289.

Jackson, A., Cavanagh, J., and Scott, J. (2003). A systematic review of manic and depressive prodromes. J. Affect. Disord. 74, 209-217.

Jansson, M., and Linton, S. J. (2006). The development of insomnia within the first year: a focus on worry. Br. J. Health Psychol. 11, 501-511.

Jenni, O. G., and Dahl, R. E. (2008). "Sleep, cognition, and emotion: a developmental view," in Handbook of Developmental Cognitive Neuroscience, 2nd Edn, eds C. A. Nelson and M. Luciana (Cambridge, MA: MIT Press), 807-817.

Johnson, E. O., Roth, T., and Breslau, N. (2006). The association of insomnia with anxiety disorders and depression: exploration of the direction of risk. J. Psychiatr. Res. 40, 700-708.

Johnson, S. L. (2005). Mania and dysregulation in goal pursuit: a review. Clin. Psychol. Rev. 25, 241-262.

Kaplan, K. A., Talbot, L. S., and Harvey, A. G. (2009). Cognitive mechanisms in chronic insomnia: processes and prospects. Sleep Med. Clin. 4, 541-548.

LeBlanc, M., Beaulieu-Bonneau, S., Mérette, C., Savard, J., Ivers, H., and Morin, C. M. (2007). Psychological and health-related quality of life factors associated with insomnia in a population-based sample. $J$. Psychosom. Res. 63, 157-166.

Levey, A. B., Aldaz, J. A., Watts, F. N., and Coyle, K. (1991). Articulatory suppression and the treatment of insomnia. Behav. Res. Ther. 29, 85-89.
Lichstein, K. L., and Rosenthal, T. L. (1980). Insomniacs' perceptions of cognitive versus somatic determinants of sleep disturbance. J. Abnorm. Psychol. 89, 105-107.

Morin, C. M., Rodrigue, S., and Ivers, H. (2003). Role of stress, arousal, and coping skills in primary insomnia. Psychosom. Med. 65, 259-267.

Najmi, S., and Wegner, D. M. (2008). "Thought suppression and psychopathology," in Handbook of Approach and Avoidance Motivation, ed. A. J. Elliott (New York, NY: Psychology Press), 447-459.

Nelson, J., and Harvey, A. G. (2002). The differential functions of imagery and verbal thought in insomnia. $J$. Abnorm. Psychol. 111, 665-669.

Nelson, J., and Harvey, A. G. (2003). Pre-sleep imagery under the microscope: a comparison of patients with insomnia and good sleepers. Behav. Res. Ther. 41, 273-284.

Nicassio, P. M., Mendlowitz, D. R., Fussell, J. J., and Petras, L. (1985). The phenomenology of the pre-sleep state: the development of the PreSleep Arousal Scale. Behav. Res. Ther. 23, 263-271.

Nolen-Hoeksema, S., Wisco, B. E., and Lyubomirsky, S. (2008). Rethinking rumination. Perspect. Psychol. Sci. 3, 400-424.

Ohayon, M. M., Caulet, M., Philip, P., Guilleminault, C., and Priest, R. G. (1997). How sleep and mental disorders are related to complaints of daytime sleepiness. Arch. Intern. Med. 157, 2645-2652.

Omvik, S., Pallesen, S., Bjorvatn, B., Thayer, J., and Nordhus, I. H. (2007). Night-time thoughts in high and low worriers: reaction to caffeineinduced sleeplessness. Behav. Res. Ther. 45, 715-727.

Ong, J. C., Shapiro, S. L., and Manber, R. (2008). Combining mindfulness meditation with cognitive-behavior therapy for insomnia: a treatmentdevelopment study. Behav. Ther. 39, 171-182.

Ree, M. J., Harvey, A. G., Blake, R., Tang, N. K. Y., and Shawe-Taylor, M. (2005). Attempts to control unwanted thoughts in the night: development of the thought control questionnaire-insomnia revised (TCQI-R). Behav. Res. Ther. 43, 985-998.

Robertson, J. A., Broomfield, N. M., and Espie, C.A. (2007). Prospective comparison of subjective arousal during the pre-sleep period in primary sleep-onset insomnia and normal sleepers. J. Sleep Res. 16, 230-238.

Robinson, M. S., and Alloy, L. B. (2003). Negative cognitive styles and stress-reactive rumination interact to predict depression: a prospective study. Cognit. Ther. Res. 27, 275-291.

Roth, T., and Ancoli-Israel, S. (1999). Daytime consequences and correlates of insomnia in the United States: results of the National Sleep Foundation Survey - II. Sleep 22, S354-S358.

Roth, T., Coulouvrat, C., Hajak, G., Lakoma, M. D., Sampson, N. A., Shahly, V., Shillington, A. C. Stephenson, J. J., Walsh, J. K., and Kessler, R. C. (2011). Prevalence and perceived health associated with insomnia based on DSM-IV-TR; International Statistical Classification of Diseases and Related Health Problems, Tenth Revision; and Research Diagnostic Criteria/International Classification of Sleep Disorders, Second Edition criteria: results from the American Insomnia Survey. Biol. Psychiatry 69, 592-600.

Scherer, K. R. (2005). What are emotions? And how can they be measured? Soc. Sci. Inf. 44, 695-729.

Schmidt, R. E., Gay, P., Ghisletta, P. and Van der Linden, M. (2010). Linking impulsivity to dysfunctional thought control and insomnia: a structural equation model. J. Sleep Res. 19, 3-11.

Schmidt, R. E., Gay, P., and Van der Linden, M. (2009). Validation of a French version of the Thought Control Questionnaire InsomniaRevised (TCQI-R). Eur. Rev. Appl. Psychol. 59, 69-78.

Schmidt, R. E., and Gendolla, G. H. E. (2008). Dreaming of white bears: the return of the suppressed at sleep onset. Conscious. Cogn. 17, 714-724.

Schmidt, R. E., Renaud, O., and Van der Linden, M. (in press). Nocturnal regrets and insomnia in elderly people. Int. J. Aging Hum. Dev.

Schmidt, R. E., and Van der Linden, M. (2009). The aftermath of rash action: sleep-interfering counterfactual thoughts and emotions. Emotion 9, 549-553.

Schmidt, R. E., and Van der Linden, M. (2011). The nocturnal return of neglected regrets: deficits in regret anticipation predict insomnia. Open. Sleep. J. 4, 20-25.

Segerstrom, S. C., Stanton, A. L., Alden, L. E., and Shortridge, B. E. (2003). A multidimensional structure for repetitive thought: what's on your mind, and how, and how much? J. Pers. Soc. Psychol. 85, 909-921.

Shin, C., Kim, J., Yi, H., Lee, H., Lee, J., and Shin, K. (2005). Relationship between trait-anger and sleep disturbances in middle-aged men and women. J. Psychosom. Res. 58, 183-189.

Sibrava, N. J., and Borkovec, T. D. (2006). "The cognitive avoidance theory of worry," in Worry and Its Psychological Disorders: Theory, Assessment, and Treatment, eds G. C. L. Davey and A. Wells (West Sussex: John Wiley \& Sons), 239-256.

Steptoe, A., O’Donnell, K., Marmot, M., and Wardle, J. (2008). Positive affect, psychological well-being, and good sleep. J. Psychosom. Res. 64, 409-415.

Stewart, J. C., Rand, K. L., Hawkins, M. A. W., and Stines, J. A. (2011) Associations of the shared and unique aspects of positive and negative emotional factors with sleep quality. Pers. Individ. Dif. 50, 609-614.

Stoia-Caraballo, R., Rye, M. S., Pan, W., Brown Kirschman, K. J., LutzZois, C., and Lyons, A. M. (2008). Negative affect and anger rumination as mediators between forgiveness and sleep quality. J. Behav. Med. 31, 478-488.

Talbot, L. S., Hairston, I. S., Eidelman, P., Gruber, J., and Harvey, A. G. (2009). The effect of mood on sleep onset latency and REM sleep in interepisode bipolar disorder. $J$. Abnorm. Psychol. 118, 448-458.

Talbot, L. S., McGlinchey, E. L., Kaplan, K. A., Dahl, R. E., and Harvey, A. G. (2010). Sleep deprivation in adolescents and adults: changes in affect. Emotion 10, 831-841.

Taylor, D. J., Lichstein, K. L., Durrence, H. H., Reidel, B. W., and Bush, A. J. (2005). Epidemiology of insomnia, depression, and anxiety. Sleep 28, 1457-1464

Taylor, F., and Bryant, R. A. (2007). The tendency to suppress, inhibiting thoughts, and dream rebound. Behav. Res. Ther. 45, 163-168.

Thomsen, D. K., Mehlsen, M. Y., Christensen, S., and Zachariae, R. (2003). Rumination - relationship with negative mood and sleep quality. Pers. Individ. Dif. 34, 1293-1301.

Vasey, M. W., and Borkovec, T. D. (1992). A catastrophizing assessment of worrisome thoughts. Cognit. Ther. Res. 16, 505-520.

Vincent, N., Cox, B., and Clara, I. (2009). Are personality dimensions associated with sleep length in a large nationally representative sample? Compr. Psychiatry 50, 158-163.

Vrana, S. R., Cuthbert, B. N., and Lang, P. J. (1986). Fear imagery and text processing. Psychophysiology 23, 247-253.

Walker, M. P., and Harvey, A. G. (2010). Obligate symbiosis: sleep and affect. Sleep Med. Rev. 14, 215-217. 
Walker, M. P., and van der Helm, E. (2009). Overnight therapy? The role of sleep in emotional brain processing. Psychol. Bull. 135, 731-748.

Watkins, E. R. (2008). Constructive and unconstructive repetitive thought. Psychol. Bull. 134, 163-206.

Watkins, E. R. (2011). Dysregulation in level of goal and action identification across psychological disorders. Clin. Psychol. Rev. 31, 260-278.

Watkins, E. R., and Moulds, M. L. (2009). Thought control strategies, thought suppression, and rumination in depression. Int. J. Cogn. Ther. 2, 235-251.

Watts, F. N., Coyle, K., and East, M. P. (1994). The contribution of worry to insomnia. Br. J. Clin. Psychol. 33, 211-220.

Wegner, D. M. (1989). White Bears and Other Unwanted Thoughts. Suppression, Obsession, and the Psychology of Mental Control. New York: Guilford Press.
Wegner, D. M. (1994). Ironic processes of mental control. Psychol. Rev. 101, 34-52.

Wegner, D. M., Wenzlaff, R. M., and Kozak, M. (2004). Dream rebound: the return of suppressed thoughts in dreams. Psychol. Sci. 15, 232-236.

Wells, A., and Davies, M. I. (1994). The thought control questionnaire: a measure of individual differences in the control of unwanted thoughts. Behav. Res. Ther. 32, 871-878.

Whiteside, S. P., and Lynam, D. R. (2001). The five factor model and impulsivity: using a structural model of personality to understand impulsivity. Pers. Individ. Dif. 30, 669-689.

Wood, A. M., Joseph, S., Lloyd, J., and Atkins, S. (2009). Gratitude influences sleep through the mechanism of pre-sleep cognitions. J. Psychosom. Res. 66, 43-48.

Wrosch, C., Bauer, I., Miller, G. E., and Lupien, S. (2007). Regret intensity, diurnal cortisol secretion, and physical health in older individuals: evidence for directional effects and protective factors. Psychol. Aging 22, 319-330.

Wrosch, C., Bauer, I., and Scheier, M. F. (2005). Regret and quality of life across the adult life span: the influence of disengagement and available future goals. Psychol. Aging 20, 657-670.

Yoo, S.-S., Gujar, N., Hu, P., Jolesz, F. A., and Walker, M. P. (2007) The human emotional brain without sleep-a prefrontal amygdala disconnect. Curr. Biol. 17, 877-878.

Zeelenberg, M., and Pieters, R. (2007). A theory of regret regulation 1.0. $\mathrm{J}$. Consum. Psychol. 17, 3-18.

Zohar, D., Tzischinsky, O., Epstein, R. and Lavie, P. (2005). The effects of sleep loss on medical residents emotional reactions to work events: a cognitive-energy model. Sleep 28 , 47-54.
Conflict of Interest Statement: The authors declare that the research was conducted in the absence of any commercial or financial relationships that could be construed as a potential conflict of interest.

Received: 31 July 2011; accepted: 08 November 2011; published online: 07 December 2011.

Citation: Schmidt RE, Harvey $A G$ and Van der Linden $M$ (2011) Cognitive and affective control in insomnia. Front. Psychology 2:349. doi: 10.3389/fpsyg.2011.00349

This article was submitted to Frontiers in Cognition, a specialty of Frontiers in Psychology.

Copyright (๑) 2011 Schmidt, Harvey and Van der Linden. This is an open-access article distributed under the terms of the Creative Commons Attribution Non Commercial License, which permits noncommercial use, distribution, and reproduction in other forums, provided the original authors and source are credited. 